\title{
Comparison Analogy Between Properties of Hypernucleus and Supernucleus with Properties of the Elementary Particles and Resonances Electroproduction by Spin Shock Waves
}

\author{
A. G. Syromyatnikov \\ Department of Physics, St. Petersburg University, Universitetskaya nab., St. Petersburg, Russia
}

Email address:

alsyromyatnikov@mail.ru

To cite this article:

A. G. Syromyatnikov. Comparison Analogy Between Properties of Hypernucleus and Supernucleus with Properties of the Elementary Particles and Resonances Electroproduction by Spin Shock Waves. International Journal of High Energy Physics. Special Issue: Breaking of Space Symmetry in the Masses Spectrum Problem. Vol. 3, No. 1-1, 2016, pp. 1-8. doi: 10.11648/j.ijhep.s.2016030101.11

Received: April 6, 2015; Accepted: April 17, 2015; Published: March 19, 2016

\begin{abstract}
It is showed generalization of discovering of the simple fact of proportionality between all elementary particles masses and 24 resonance masses in one side and nuclei weights in another side with some constant coefficient to all-known elementary particles and resonances masses. For resonances that linear dependence led to elementary particles type when electric charge of ions is defined by the same charge of muons. This is done within the frame of the "eight spin shock wave" model, which is based on an accurate solution of the Maxwell equations for a dust-like medium of charged particles in the flat space. Parameters of the group, such as Euler's angles and Lorentz boosts, are all localized. In this way, they become functions of space coordinates and time. As a result, it is found that the Lorentz boost's argument satisfies the linear wave equation. For small angles, the "eight spin" model is similar to the fundamental O(3) model. It is stated some analogy between properties of hypernucleus and supernucleus with properties of elementary particles and resonances electroproduction by spin shock waves (SSW) in beam ions. In particularly theoretically for all hypernucleus it very may be possible that $\Lambda$ interacts with two $\mathrm{NN}$ from the external level of nucleus core. The ions list for resonances begin by $(n, p)$ nucleus, on the first hypernucleus decay channel - on $\mathrm{D}_{2}$. For $\mathrm{He}_{4}$ in the $\tau$ - atom $\mathrm{m}_{\mathrm{\tau}} \mathrm{M}^{-1}=0.475$ is near 0.41 is seen through the lepton universality as atom on the outer shell of which, instead of electron - a heavy lepton - tauon etc. In this approach neutral Higgs`s boson with it mass $125 \mathrm{GeV}$ connected with nucleus from the island of stability, so as neutral $\mathrm{Z}$ boson - to $\mathrm{U}-238$. On this set of quarks distribution their dilaton currents conservation is confirmed. Theoretically nuclei weights are defined by according to dilaton quark - lepton $\mathrm{X}$ - structure identified with jumps features of the quantum Green function. Whereas the definition of a value of the ratio between the masses of particles and nuclei requires taking into account the contribution of SSW C-number components of classical interact.
\end{abstract}

Keywords: Dilaton or Scale Symmetry, Dilaton Quark - Lepton X - Structure, Particles Photoproduction in Hypernucleus, Spin Shock Wave

\section{Introduction}

Elementary particles and resonance production in high energy processes is characterized by scaling, when there is dilaton currents conservation low. It leads to fundamental definition of lepton number as a quantum dilaton number (see for example [1]) such as lepton generations are distinguished on masses only. In another side in the analogy it may be possible dilaton quarks currents conservation too.
In the work [2] it is showed in possibility processes of elementary particles and resonance electroproduction on a threshold of spin shock-waves forming [1] in accelerated beams of ions of some stable chemical elements. In that case it may be possible start from the simple fact of proportionality between all elementary particles masses and 24 resonance masses [3-5] in one side and nuclei masses in another side with some constant coefficient to all-known elementary particles and resonances mass (see list [4]).

In that unexpected repeating of the whole by a fragments 
spectrum in 3 cases for particles and in 11 cases for resonances fit took admissible doubling of this calculated dilatation coefficient by means an ion electric charge doubling (in possibility processes of their electroproduction on a threshold of spin shock-waves (see [6]) forming [1] in accelerated beams of ions of some stable chemical elements; their photoproduction in hypernucleus and supernucleus also with one or two particles see [7-10]. Considerations of all known resonances from particles list [4] according to underlying pages show that a number of this cases electric charge doubling more 55 percent. Fundamentally in this case that $\mathrm{W}^{+}$ boson of electroweak interaction in recalculating on mass directly join to a border of chemical elements stability through $\mathrm{Bi}-209$.

If so it be on more number of resonances then there mass spectrum are defined by chemical elements mass spectrum, relation charge to mass of electron, speed of light in vacuum and calculated threshold value spin shock-wave. Chemical elements (CE) masses are defined by a dilaton quark - lepton $\mathrm{X}$ - structure jump features $[1,2]$.

According to [2] elementary particles and resonances group to every electroproduction sets on a mass with a small dispersion 78 and $70 \mathrm{MeV}$ accordingly. In particularly it is $\mathrm{SU}(3)$ baryon octet, distinguishing from the well-known by two baryons from hyperons triplet $\Delta^{0,+,++}$ instead of two nucleons, SU(3) singlet $\Omega^{-}$et cetera. Two nucleons essentially distinguish on their mass from the other particles from octet included to the triplet with one from the hyperon triplet $\Delta^{0,+,++}$. On the fact of proportion (see below) between all-known elementary particles masses and resonances masses [3-5] in one side and nuclei masses in another side of which is stated it may be possible that quark spectrum from particles and resonances forming on nuclei destruction also will be homogeneous distribution as a Fermi distribution.

Actually according to [2] quark distributions on electroproduction set all-known elementary particles and resonances are homogeneous as Fermi distributions. Moreover the number of sets of particles 1, 2, 3, 4, 8 formed Fibonacci numbers series. As known Fibonacci numbers takes place in quality of the solution of a task about degeneration of a level in the dual model. It supposed the fact that there we have filling of any level from 18 degree of freedom on a number of quarks. Standard deviation on a number of 18 quarks for particles and on totality particles and quarks whole is smaller than 2. It means that the deviation from the homogeneity of the quark distribution turned out far from an observables border for the single quark.

Dilatonsupersymmetry thesis [1] about 18 degree of freedom for quark system as freedom fermions system in Veil - Cartan space - time is supposed exactly [2].

By oneself fact of homogeneity of a quark distributions show dilaton quark quantum number conservation (see [1]) as independent from kind of any electroproduction sets.

In the work [2] it is stated that all discovered 46 elementary particles and 121 resonances all group in electroproduction sets for every ion kind on that occur their electroproduction as in [1] or more detailed on channels of hypernucleus ${ }_{\Omega}^{A} C E$ or
${ }_{\Omega \Omega}^{A} C E$ and supernucleus ${ }_{\Lambda}^{A} C E$ or ${ }_{\Lambda \Lambda}^{A} C E$ photoproduction $(\mathrm{CE}$ - chemical element nuclei, $A$-the hypernucleus or supernucleus mass, $\Omega$ - hyperon, $\Lambda$ - elementary particle or resonance). In this approach neutral Higgs`s boson with it mass $125 \mathrm{GeV}$ connected with nucleus from the island of stability, so as neutral Z boson - to U -238 .

In this work we study comparison analogy between properties of hipernucleus and supernucleus with properties of electroproduction of elementary particles and resonances by spin shock waves in beam ions. The work constructed by the following way. In the first it will be given statistic dates [2] for the linear dependence between series of 50 elementary particles masses and 121 resonances masses spectrum and corresponding nuclei atomic weights. Then show the analogy between properties of hipernucleus and supernucleus with properties of electroproduction of elementary particles and resonances by spin shock waves in beam ions.

\section{Statistics}

It is stated [2] that there is 0.999952 correlations coefficient between series of 50 elementary particles masses spectrum and corresponding nuclei atomic weights. It is direct non statatistics dependence. So more this linear dependence of type $\mathrm{m}_{\mathrm{EP}}=\mathrm{aM}_{\mathrm{N}} \mathrm{Z}^{-1}+\mathrm{b}$ with $\mathrm{W}^{+}$boson of electroweak interaction in recalculating on At -210 and $Z^{0}$ boson on $\mathrm{U}-238$ are characterized by standard deviation $\mathrm{a}=0.4114 \pm$ $0.00058, \mathrm{~b}=16.32 \pm 25.18 \mathrm{MeV}$. Theoretically $\mathrm{a}=0.411$.

There is 0.99872 correlations coefficient between series of 121 resonances masses spectrum and corresponding nuclei atomic weights. Linear dependence of type $m_{\text {RES }}=\mathrm{aM}_{\mathrm{N}} \mathrm{Z}^{-1}+$ $\mathrm{b}_{\text {res }}$ is characterized by standard deviation $\mathrm{a}=0.4044 \pm 0.0019$, $\mathrm{b}_{\text {res }}=67.4 \pm 17.3 \mathrm{MeV}$.

In whole there is 0.999882 correlations coefficient between series of 50 elementary particles and 121 resonances masses spectrum and corresponding nuclei atomic weights. Linear dependence of type $\mathrm{m}=\mathrm{aM}_{\mathrm{N}} \mathrm{Z}^{-1}+\mathrm{b}$ is characterized by standard deviation $\mathrm{a}=0.411014 \pm 0.00050, \mathrm{~b}=22.9 \pm 12.0$ $\mathrm{MeV}$. Theoretically a $=0.411$. These high correlations do not be any simple coincidence [2].

2. On a possibility of the particles and resonances production in a spin shock - wave of an accelerated ion beam.

In high energy physics the all information about system defined by features of scattering amplitude on masses of particles ore by features on an angular momentum. Masses of chemical elements down to a border of their stability according to dilaton quark - lepton $\mathrm{X}$ - structure [1] identified with jumps features of the quantum Green function. Fundamentally that features on angular momentum in Regge theory [3] follow direct from dynamics but only with taking into account of a space-time torsion, such as spin angular momentum is a source of torsion field [1]. In particular it leads to features on a spin of a jump form that is directly to spin s hock-waves (SSW) with spin-flip effects on front of the wave. I give the idea of possibility of particles and resonances production by spin shock-waves $[1,2]$. In one side in beam ions has not scattering between oneself. SSW production 
course by features of beam collective electromagnetic field. In other side ions directly participates in collective self-consistent electromagnetic field spin-flip effect. For a clarification of this question it was considering a problem of spin shock-waves production by ions beam. Threshold of spin shock-waves forming on an accelerating voltage $U_{\mathrm{SSw}}$ is defines by an ion electric charge $Z$ and his mass $M$ so as 210 $\mathrm{keV}$ for an electron beam [1]: $\mathrm{ZU}_{\mathrm{SSW}}=\mathrm{M} \cdot 210 \mathrm{keV} /(511 \mathrm{keV})$ $=0.411$. Theoretically the definition of this value of the ratio between the masses of particles and nuclei requires taking into account the contribution of SSW C-number components of classical interact. In all cases a spin shock-waves forming inside an accelerating gap. It can be allowed that this threshold voltage will do for particles and resonances with mass $M$ production.

Below on table 1 all discovered 46 elementary particles and in table 2 all group in some sets for every ion kind on that occur their electroproduction as in [1] or more detailed on channels of hypernucleus ${ }_{\Omega}^{A} C E$ or ${ }_{\Omega \Omega}^{A} C E$ and supernucleus ${ }_{\Lambda}^{A} C E$ or ${ }_{\Lambda \Lambda}^{A} C E$ photoproduction (CE - chemical element nuclei, A -the hypernucleus or supernucleus mass, $\Omega$ hyperon, $\Lambda$ - elementary particle or resonance). In this approach neutral Higgs's boson with it mass $125 \mathrm{GeV}$ connected with nucleus from the island of stability, so as neutral $\mathrm{Z}$ boson - to $\mathrm{U}-238$.

According to table 3 in average the every set consist of 18 quarks. The distribution on resonances also is characterized every set in average from 18 quarks in limits of the permissible dispersion.

Below in table 3 give collections of sets of table 1 in the distribution on a number of quarks with doublet nucleons addition. All it is in direct accordance with thesis (see [1]) dilatonsupersymmetry about 18 degree of freedom for quark system as freedom fermions system in Veil - Cartan space time (see $[1,11])$.

Table 1. All discovered 46 elementary particles group in some sets for every ion kind on that occur their electroproduction.

\begin{tabular}{|c|c|c|c|}
\hline $\mathbf{C E}^{1}$ & $\mathbf{A}^{2}$ & $\mathbf{B}^{3}$ & Particles \\
\hline $\mathrm{p}^{+}$ & 3 & \pm 32 & $\mathrm{~K}^{+}[493,7] \mathrm{K}_{\mathrm{LS}}^{0}[497,6] \eta^{0}[547,8]$ \\
\hline \multirow[t]{3}{*}{$\mathrm{He}_{3}$} & 8 & \pm 68 & $\Delta^{0}, \Delta^{+}[1232] \Lambda^{0}[1232] \Sigma^{+}[1189,4]$ \\
\hline & & & $\Sigma^{0}[1192,5] \Sigma[1197,4] \Xi^{0}[1315]$ \\
\hline & & & $\Xi[1321]$ \\
\hline $\mathrm{He}_{3}$ & 3 & \pm 170 & $\mathrm{p}^{+} \mathrm{n}[938] \Delta^{++}[1232]$ \\
\hline $\mathrm{He}_{3}$ & 1 & - & $\varphi[1019]$ \\
\hline $\mathrm{He}_{4}$ & 1 & - & $\Omega[1672]$ \\
\hline $\mathrm{Li}_{7}$ & 1 & - & $\Omega_{\mathrm{c}}^{0}[2698]$ \\
\hline $\mathrm{B}_{11}^{2+}$ & 1 & - & $\mathrm{D}[1870]$ \\
\hline \multirow[t]{2}{*}{$\mathrm{N}_{14}^{2+}$} & 6 & \pm 110 & $\Lambda_{\mathrm{c}}^{+}[2284] \Lambda_{\mathrm{c}}^{0}[2274] \Xi_{\mathrm{c}}^{+}[2466]$ \\
\hline & & & $\Xi_{\mathrm{c}}^{0}[2472] \Lambda_{\mathrm{c}}^{-}[2284] \Xi_{\mathrm{c}}^{-}[2466]$ \\
\hline $\mathrm{C}_{12}{ }^{2+}$ & 2 & \pm 51 & $\mathrm{D}^{*}[2010] \mathrm{D}_{\mathrm{s}}^{*}[2112]$ \\
\hline $\mathrm{Be}_{9}$ & 1 & - & $\eta_{\mathrm{c}}^{\prime}[3592]$ \\
\hline $\mathrm{O}_{16}{ }^{2-}$ & 2 & \pm 68 & $\mathrm{~J} / \psi[3097] \eta_{\mathrm{c}}[2980]$ \\
\hline $\mathrm{B}_{11}$ & 2 & \pm 42 & $\Psi^{\prime}[3685] \Psi^{\prime \prime}[3768]$ \\
\hline $\mathrm{C}_{12}$ & 1 & - & $\Psi^{\prime \prime \prime}[4415]$ \\
\hline $\mathrm{N}_{14}$ & 3 & \pm 45 & $\mathrm{~B}^{*}[5325] \mathrm{B}_{\mathrm{s}}^{0}[5366] \mathrm{B}_{\mathrm{s}}^{*}[5415]$ \\
\hline \multirow[t]{3}{*}{$\mathrm{O}_{15}$} & 7 & \pm 104 & $\Lambda_{\mathrm{b}}^{0}[5624] \mathrm{B}_{\mathrm{s} 1}[5830] \mathrm{B}_{\mathrm{s} 2}[5840]$ \\
\hline & & & $\Xi_{\mathrm{b}}[5629,6] \mathrm{B}_{1}[5721] \bar{\Lambda}_{b}^{0}[5624]$ \\
\hline & & & $\bar{\Xi}_{b}[5629,6]$ \\
\hline $\mathrm{O}_{16}$ & 2 & 0 & $\mathrm{~B}_{\mathrm{c}}^{+}[6277] \mathrm{B}_{\mathrm{c}}^{-}[6277]$ \\
\hline $\mathrm{Mg}_{24}$ & 1 & - & $\mathrm{Y}[9459,7]$ \\
\hline $\mathrm{Al}_{27}$ & 2 & \pm 166 & $Y^{\prime}[10018] Y^{\prime \prime}[10350]$ \\
\hline $\mathrm{Si}_{28}$ & 1 & - & $Y^{\prime \prime \prime}[10573]$ \\
\hline average & & $\pm 78 \mathrm{MэB}$ & \\
\hline
\end{tabular}

${ }^{1}$ Ions of chemical elements (electric charge +1 or -1 is omitted)

${ }^{2}$ Number of particles in the set

${ }^{3}$ Standard deviation on distinguish between particles masses, $\mathrm{MeV}$

Table 2. All discovered 121 resonances group in some sets for every ion kind on that occur their electroproduction.

\begin{tabular}{llll}
\hline $\mathbf{C E}^{\mathbf{1}}$ & $\mathbf{A}^{\mathbf{2}}$ & $\mathbf{B}^{\mathbf{3}}$ & Resonances \\
\hline $\mathrm{D}_{2}$ & 4 & $748 \pm 145$ & $\rho^{+}[770] \mathrm{K}^{*}[892] \omega[783]$ \\
771 & 9 & $1102 \pm 115$ & $\eta[548]$ \\
& & $\mathrm{f}_{0}[980] \mathrm{a}_{0}[980] \varphi[1019]$ \\
$\mathrm{He}_{3}$ & & $\eta_{1}[1170] \mathrm{a}_{1}[1230] \mathrm{b}_{1}[1230]$ \\
1157 & & $\mathrm{f}_{1}[1282] \mathrm{K}_{1}[1270] \mathrm{f}_{2}[1270]$ \\
& 6 & $\Delta_{0}[1230] \Sigma^{0}[1193] \Sigma^{+}[1190]$ \\
$\mathrm{Li}_{7}{ }^{2+}$ & 4 & $\Sigma[1197] \Xi^{0}[1314] \Xi^{0}[1322]$ \\
1338 & & $\eta[1294] \pi[1300] \mathrm{a}_{2}[1320]$ \\
& 5 & $1320 \pm 35$ & $\mathrm{f}_{0}[1370]$ \\
\hline
\end{tabular}




\begin{tabular}{|c|c|c|c|}
\hline $\mathrm{CE}^{1}$ & $\overline{A^{2}}$ & $\mathbf{B}^{3}$ & Resonances \\
\hline & & & $\mathrm{K}_{2}^{*}[1430] \mathrm{f}_{1}[1426]$ \\
\hline & 6 & $1533 \pm 75$ & $\rho[1450] \eta[1476] \mathrm{f}_{0}[1500]$ \\
\hline $\mathrm{He}_{4}$ & & & $\mathrm{f}_{2}[1525] \pi_{1}[1600] \pi_{2}[1645]$ \\
\hline \multirow[t]{6}{*}{1542} & & & $\Sigma[1385] \Sigma[1530] \Lambda[1405]$ \\
\hline & 4 & $1468 \pm 126$ & $\omega[1650]$ \\
\hline & 10 & $1710 \pm 50$ & $\pi_{2}[1670] \omega_{3}[1670] \varphi[1680]$ \\
\hline & & & $\mathrm{K}^{*}[1680] \rho_{3}[1700] \mathrm{f}_{0}[1710]$ \\
\hline & & & $\mathrm{K}_{3}[1770] \pi[1800] \chi[1835]$ \\
\hline & & & $\mathrm{K}_{2}[1820]$ \\
\hline $\mathrm{Be}_{9}{ }^{2+}$ & 2 & & $\varphi_{3}[1850] \pi_{2}[1880]$ \\
\hline \multirow[t]{7}{*}{1724} & 7 & $1734 \pm 65$ & $\Sigma[1650] \Sigma[1670]$ \\
\hline & & & $\Xi[1690]$ \\
\hline & & & $\Sigma[1750] \Lambda[1800] \Lambda[1810]$ \\
\hline & & & $\mathrm{K}_{2}[1770]$ \\
\hline & 3 & $1840 \pm 40$ & $\Lambda[1820] \Xi[1820] \Lambda[1890]$ \\
\hline & & & $\Sigma[1915] \Sigma[1940]$ \\
\hline & 8 & $1968 \pm 45$ & $\Xi[1950] \Sigma[2030]$ \\
\hline \multirow{6}{*}{$\begin{array}{l}\mathrm{B}_{11}^{2+} \\
1931\end{array}$} & & & $\Xi[2030] \Lambda[2100]$ \\
\hline & & & $\mathrm{f}_{2}[1950] \mathrm{f}_{2}[1959]$ \\
\hline & & & $\Lambda[2110] \mathrm{a}_{4}[2040] \mathrm{D}^{*}[2007]$ \\
\hline & 8 & $2039 \pm 37$ & $\mathrm{D}^{*}[2010] \mathrm{f}_{2}[2010] \mathrm{f}_{4}[2050]$ \\
\hline & & & $\mathrm{K}_{4}^{*}[2045] \varphi[2170]$ \\
\hline & 3 & $2283 \pm 55$ & $\Sigma[2250] \Omega^{-}[2250] \Lambda[2350]$ \\
\hline \multirow{3}{*}{$\begin{array}{l}\mathrm{C}_{12}{ }^{2+} \\
2145\end{array}$} & & & $\mathrm{f}_{2}[2300] \mathrm{f}_{2}[2340]$ \\
\hline & 6 & $2280 \pm 80$ & $\mathrm{D}^{*}{ }_{00}[2317] \mathrm{D}_{2}^{*}[2160]$ \\
\hline & & & $\mathrm{D}_{\mathrm{s} 1}[2460] \mathrm{D}_{1}[2420]$ \\
\hline \multirow{4}{*}{$\begin{array}{l}\mathrm{Li}_{7} \\
2676\end{array}$} & & & $\Sigma_{\mathrm{c}}[2520] \Lambda_{\mathrm{c}}[2595]$ \\
\hline & 6 & $2582 \pm 43$ & $\Lambda_{\mathrm{c}}[2625] \Xi_{\mathrm{c}}[2645]$ \\
\hline & & & $\mathrm{D}_{\mathrm{s} 1}[2536] \mathrm{D}_{\mathrm{s} 2}[2572]$ \\
\hline & & & $\Xi[3080] \Xi_{\mathrm{c}}[2800]$ \\
\hline $\mathrm{O}_{15^{2-}}$ & & & $\Lambda_{\mathrm{c}}[2880] \Lambda_{\mathrm{c}}[2940]$ \\
\hline \multirow[t]{2}{*}{2868} & 7 & $2916 \pm 70$ & $\Xi[2980]$ \\
\hline & & & $\eta_{c}(1 \mathrm{~S} 1)[2980] \mathrm{J} / \psi[3097]$ \\
\hline \multirow{3}{*}{$\begin{array}{l}\mathrm{Be}_{9} \\
3447\end{array}$} & 5 & & $\chi_{\mathrm{co}}[3415] \chi_{\mathrm{cl}}(1 \mathrm{P})[3511]$ \\
\hline & & $3502 \pm 60$ & $\chi_{c 22}(1 \mathrm{P})[3556] \mathrm{h}_{\mathrm{c}}(1 \mathrm{P})[3525]$ \\
\hline & & & $\eta_{c}(2 S)[3637]$ \\
\hline $\begin{array}{l}\mathrm{F}_{19^{2-}} \\
3639\end{array}$ & 1 & & $\psi(2 s)[3626]$ \\
\hline $\mathrm{B}_{11}$ & 3 & $3894 \pm 140$ & $\psi[3770] \chi[3872] \psi[4040]$ \\
\hline $\mathrm{Na}_{23}{ }^{2+}$ & & & $\psi^{\prime \prime \prime}[4160] \chi[3872]$ \\
\hline $\begin{array}{l}4348 \\
\mathrm{Mg}_{24.3}\end{array}$ & 3 & $4280 \pm 130$ & $\psi[4415]$ \\
\hline $\begin{array}{l}\mathrm{Mg}_{24.3} \\
9298\end{array}$ & 1 & & $\gamma[9460]$ \\
\hline \multirow{2}{*}{$\begin{array}{l}\mathrm{Cr}_{52}{ }^{2+} \\
9925\end{array}$} & 3 & $0030+80$ & $\chi_{\mathrm{bo}}(1 \mathrm{P})[9856] \chi_{\mathrm{b} 2}(1 \mathrm{P})[9912]$ \\
\hline & 3 & $9930 \pm 89$ & $\gamma(2 S)[10023]$ \\
\hline \multirow{3}{*}{$\begin{array}{l}\mathrm{Al}_{27} \\
10306\end{array}$} & & & $\gamma(1 \mathrm{D})[10163]$ \\
\hline & 4 & $10251 \pm 80$ & $\chi_{b 0}(2 P)[10232,5]$ \\
\hline & & & $\chi_{b 1}(2 S)[10255,4] \gamma(3 S)[10355]$ \\
\hline \multirow{3}{*}{$\begin{array}{l}\mathrm{Si}_{28} \\
10688 \\
\mathrm{Ni}_{59}{ }^{2+} \\
11201\end{array}$} & 2 & $10720 \pm 140$ & $\gamma(4 \mathrm{~S})[10580] \gamma[10860]$ \\
\hline & 1 & & $\gamma[1]$ \\
\hline & 1 & & $\gamma[11$ \\
\hline average & & $\pm 70 \mathrm{MeV}$ & $\begin{array}{l}\text { Average distinguish between electroproduction threshold and set centre } \\
28 \pm 85 \mathrm{MeV}\end{array}$ \\
\hline
\end{tabular}

${ }^{1}$ Number of particles in the set

${ }^{2}$ Standard deviation on distinguish between particles masses, $\mathrm{MeV}$

${ }^{3}$ Ions of chemical elements (electric charge +1 or -1 is omit) Electroproduction threshold, $\mathrm{MeV}$

\section{Discussion}

Displacement $b_{\text {res }}$ for resonances is more than for particles. In another side it may be possible leads to the same type as for particles when electric charge of ions is defined by the same charge of muons: $b_{\text {res }}=24.5 \mathrm{MeV}$. There is a lepton $\mathrm{e}^{-}-\mu^{-}$ universality.

According table 1, 2 elementary particles and resonances group to every electroproduction sets on a mass with a small dispersion 78 and $70 \mathrm{MeV}$ accordingly. In particularly it is 
SU(3) baryon octet, distinguishing from the well-known by two baryons from hyperons triplet $\Delta^{0,+,++}$ instead of two nucleons, $\mathrm{SU}(3)$ singlet $\Omega^{-}$et cetera. Two nucleonsessentially distinguish on their mass from the other particles from octet included to the triplet with one from the hyperon triplet $\Delta^{0,+,++}$.

On the fact of proportion between all-known elementary particles masses and resonances masses [2-4] in one side and nuclei masses in another side of which is stated it may be possible that quark spectrum from particles and resonances forming on nuclei destruction also will be homogeneous distribution asa Fermi distribution.

Actually according to table 3, 4 quark distributions on electroproduction sets all-known elementary particles and resonances are homogeneous as Fermi distributions. Moreover the number of sets of particles 1, 2, 3, 5, 8 formed Fibonacci numbers series. As known Fibonacci numbers takes place in quality of the solution of a task about degeneration of a level in the dual model. It supposed the fact that there we have filling of any level from 18 degree of freedom on a number of quarks.

Standard deviation on a number of 18 quarks for particles and on totality particles and quarks whole is smaller then 2 . It means that the deviation from the homogeneity of the quark distribution turned out far from an observables border for the single quark.

Table 3. Quarks distribution on sets of 46 particles [4] electroproduction in ion beams.

\begin{tabular}{llllllll}
\hline Number of particles in set & $\mathbf{1}$ & $\mathbf{2}$ & $\mathbf{3}$ & $\mathbf{6}$ & $\mathbf{7}$ & $\mathbf{8}$ & 1 \\
\hline Number of sets & 8 & 5 & 3 & 1 & 1 & 18 & 24 \\
Total number of quarks & 18 & 20 & 21 & 18 & -1 & -1 & $3.5 \sigma$ \\
Deviation from the average & -1 & 1 & 2 & number of quarks & $19.0 \pm 1.42$ \\
\hline
\end{tabular}

Table 4. Quarks distribution on sets of 121 resonances and 46 particles electroproduction in ion beams.

\begin{tabular}{|c|c|c|c|c|c|c|c|c|c|}
\hline 1 & & 2 & 3 & 4 & 5 & 6 & 7 & 8 & 9 \\
\hline \multicolumn{2}{|l|}{$\mathrm{N}^{1}$} & $\begin{array}{l}1 \\
1-2\end{array}$ & 2 & 3 & 3 & 4 & 4 & 5 & 6 \\
\hline \multicolumn{2}{|l|}{$\mathrm{N}^{2}$} & $3-2$ & & 2 & 3 & 1 & 3 & 2 & 1 \\
\hline \multicolumn{2}{|l|}{$\mathrm{T}^{3}$} & $\begin{array}{l}6,8 \\
14\end{array}$ & & 18 & 18 & 14 & 24 & 20 & 16 \\
\hline \multirow[t]{2}{*}{$D^{4}$} & & -4 & & 0 & 0 & -4 & $\sim 2.5 \sigma$ & 2 & -2 \\
\hline & 10 & 11 & & 12 & 13 & 14 & 15 & 16 & 17 \\
\hline $\mathrm{N}^{2}$ & 2 & 1 & & 1 & 1 & 1 & 1 & 1 & 1 \\
\hline $\mathrm{T}^{3}$ & 24 & 18 & & 17 & 20 & 19 & 22 & 18 & 20 \\
\hline $\mathrm{D}^{4}$ & $2.5 \sigma$ & 0 & & -1 & 2 & 1 & 4 & 0 & 2 \\
\hline \multicolumn{8}{|c|}{ Standard deviation on the number of quarks } & 18.0 & \\
\hline \multicolumn{8}{|c|}{$\begin{array}{l}\text { Standard deviation on the number of quarks for all particles from table 4. (apart from the octet) and resonances [4] No. 2-3, } \\
4-6,8,9,11-14,16-17\end{array}$} & 18.0 & \\
\hline
\end{tabular}

${ }^{1}$ Number of particles in set

${ }^{2}$ Number of sets

${ }^{3}$ Total number of quarks

${ }^{4}$ Deviation from the average

By oneself fact of homogeneity of a quark distributions show a dilaton quark quantum number conservation (see [1]) as independent from kind of an electroproduction sets.

\section{Phenomenology}

A hypernucleus is a nucleus which contains at least one hyperon in addition to nucleons. The first was discovered by M. Danysz and J. Pniewski in1952. Since the strangeness quantum number is conserved by the strong and electromagnetic interactions, at least hypernuclei containing the lightest hyperon, the Lambda, live long enough to have sharp nuclear energy levels. Therefore they offer opportunities for nuclear spectroscopy, as well as reaction mechanism study and other types of nuclear physics (hypernuclear physics). Their physics is different from that of normal nuclei because a hyperon, having a different value of the strangeness quantum number, can share space and momentum coordinates with the usual four nucleons that can differ from each other in spin and isospin. The ground state of ${ }_{\Lambda}^{5} \mathrm{He}$, for example, must resemble helium-4 more than it does helium-5 or lithium-5 and must be stable, except for the weak decay of the Lambda. Sigma hypernuclei have been sought with apparent success.

Hypernuclei can be made by a nucleus capturing a Lambda or $\mathrm{K}$ meson and boiling off neutrons in a compound nuclear reaction, or, perhaps most easily, by the direct strangeness exchange reaction $\mathrm{K}+$ nucleus $\rightarrow \pi+$ hypernucleus and by channels of hypernucleus ${ }_{\Omega}^{A} C E$ or ${ }_{\Omega \Omega}^{A} C E$ and supernucleus ${ }_{\Lambda}^{A} C E$ or ${ }_{\Lambda \Lambda}^{A} C E$ photoproduction (see, for example, [8]).

Nuclei are abundant in the universe, but antinuclei that are heavier than the antiproton have been observed only as products of interactions at particle accelerators [10]. Collisions of heavy nuclei at the Relativistic Heavy-Ion Collider (RHIC) at Brookhaven National Laboratory (BNL) briefly produce hot and dense matter that has been interpreted as quark gluon plasma (QGP) with an extremely high energy density. This plasma contains roughly equal numbers of quarks and antiquarks. As a result of the high energy density of the QGP phase, many strange-antistrange (s s) quark pairs are liberated from the quantum vacuum. The plasma cools and 
transitions into a hadron gas, producing nucleons, hyperons, mesons, and their antiparticles. Nucleons (protons and neutrons) contain only up and down valence quarks, while hyperons $(\Lambda, \Sigma, \Xi, \Omega)$ contain at least one strange quark in its 3 -quark valence set. A hypernucleus is a nucleus that contains at least one hyperon in addition to nucleons. All hyperons are unstable, even when bound in nuclei. The lightest bound hypernucleus is the hypertriton $\left({ }_{\Lambda}^{3} H\right)$, which consists of a $\Lambda$ hyperon, a proton, and a neutron. Of course, it may be possible lightest hypernucleus and antinuclei production on heavy nuclei collisions, as it presented in the observation of an antimatter hypernucleus [10]: analysis yields $70 \pm 17$ antihypertritons $\left(\frac{3}{\Lambda} H\right)$ and $157 \pm 30$ hypertritons $\left({ }_{\Lambda}^{3} H\right)$ in $\mathrm{Au}+$ Au collisions.

Production of antinuclei: Models of heavy-ion collisions have had good success in explaining the production of nuclei by assuming that a statistical coalescence mechanism is in effect during the late stage of the collision evolution (see [10]) Antinuclei can be produced through the same coalescence mechanism, and are predicted to be present in cosmic rays. An observed high yield could be interpreted as an indirect signature of new physics, such as Dark Matter (see [10]). Heavy-ion collisions at RHIC provide an opportunity for the discovery and study of many antinuclei and antihypernuclei up to $\frac{3}{\Lambda} H$. The hyperon-nucleon (YN) interaction is of fundamental interest in nuclear physics and nuclear astrophysics [10]. These hypernucleus calculations includes the parameters of the distribution of nucleons in nuclei core dates taken from the $\mu$ (muons) - atom dates and electron scattering. For example, the $\mathrm{YN}$ interaction plays an important role in attempts to understand the structure of neutron stars. Depending on the strength of the YN interaction, the collapsed stellar core could be composed of hyperons, of strange quark matter, or of a kaon condensate. While the hyperons or strange particles inside a dense neutron star would not decay because of local energy constraints, free hypernuclei decay into ordinary nuclei with typical lifetimes of a few hundred picoseconds, which is still thirteen orders of magnitude longer than the lifetimes of the shortest-lived particles. The life time of a hypernucleus depends on the strength of the YN interaction. Therefore, a precise determination of the lifetime of hypernuclei provides direct information on the YN interaction strength (see [10]). The experiment was carried out by the STAR collaboration. The best fit values (from $\chi^{2}$ minimization) are $\mathrm{m}\left({ }_{\Lambda}^{3} \mathrm{H}\right)=2.989 \pm 0.001 \pm 0.002 \mathrm{GeV} / \mathrm{c}^{2}$ and $\mathrm{m}\left(\frac{3}{\Lambda} H\right)=2.991 \pm 0.001 \pm 0.002 \mathrm{GeV} / \mathrm{c}^{2}$. The systematic error of $2 \mathrm{MeV} / \mathrm{c}^{2}$ arises from well-understood instrumental effects [10].A lifetime $\tau\left({ }_{\Lambda}^{3} H\right)$ is $182 \pm 27$ ps. The ${ }_{\Lambda}^{3} H$ lifetime measurements to date are not sufficiently accurate, but measurement [10] is consistent with a calculation using a phenomenological $3 \Lambda \mathrm{H}$ wave function, and is also consistent with a more recent three-body calculation using a more modern description of the baryon-baryon force. The result [10] is also comparable to the lifetime of free $\Lambda$ particles within the uncertainties, and is statistically competitive with the earlier experimental measurements.

Below in table 5 show the analogy between properties of hypernucleus and supernucleus with properties of elementary particles and resonances electroproduction by spin shock waves in beam ions. There is a special question: can we conclude from these data that is either on the ratio

$$
\frac{m_{E P, \text { Res }}}{M_{\text {Nucleous }}}=0.412 \ldots
$$

Table 5. The analogy between properties of the proportionality between all-known elementary particles masses and resonance masses (see list [4]) in one side and nuclei masses in another side with some constant coefficient and properties of hipernucleus and supernucleus.

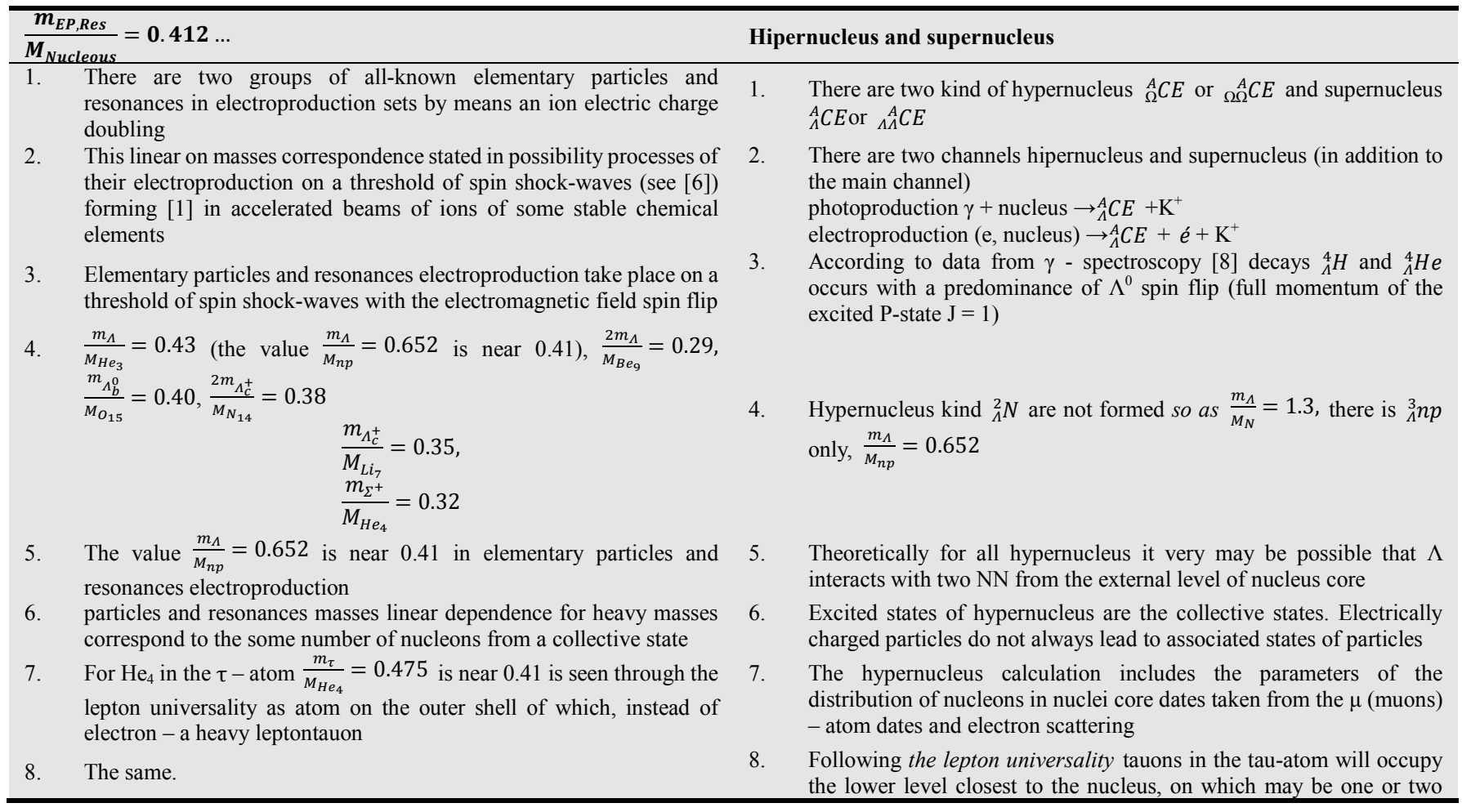




\begin{tabular}{|c|c|}
\hline$\frac{m_{E P, \text { Res }}}{M_{\text {Nucleous }}}=0.412 \ldots$ & Hipernucleus and supernucleus \\
\hline \multirow[b]{2}{*}{ 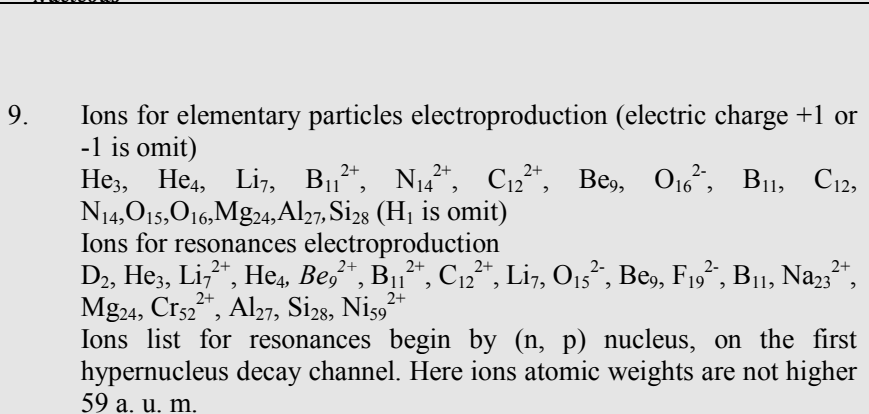 } & $\begin{array}{l}\text { particles. Hyperons mass near to the mass of the tauon, likely will also } \\
\text { be able to hold the most one- and two particles level of the lower shell. } \\
\text { What is in hypernucleuscase. }\end{array}$ \\
\hline & $\begin{array}{l}\text { Hypernucleus kind }{ }_{\Lambda}^{2} N \text { is not formed. From }{ }_{\Lambda}^{3} \mathrm{NN} \text { here is }{ }_{\Lambda}^{3} \mathrm{D}_{2} \text { only. } \\
\text { In hypernucleus of heavy } \mathrm{CE} \text { the bound energy is saturated. In decay } \\
\text { of such hypernucleus can be anything. }\end{array}$ \\
\hline
\end{tabular}

\section{Dilaton Quark-Lepton X - Structure Theory}

According to quark-lepton $\mathrm{X}$ - structure theory [1] there are following components of nucleus - nucleons, $\alpha$-particles and the 9 nucleons compound $\left(\mathrm{Be}_{9}\right)$ with one muon - from which you can compile any stable nucleus. In the hypernucleus hyperon (with the mass near the tauon mass) interacts with these components. In addition, the mass of any stable nucleus is equal to integer number of the tauon mass $(\mathrm{SD}= \pm 0.5 \mathrm{a}$. $\mathrm{u}$. $\mathrm{m}$.). Therefore, the relationship of the masses of all the particles also defined in integer numbers.

In [1] the notion of spin resolved by identifying the chemical elements $(C E)$ tables Mendeleev resonant $\mathrm{X}$-structure applicable to the atom as system a large number of weak related items. X-structure, as a representation of a generic key mass transformation of the similarity of a dilatation-subgroup includes scale sprains, built on the effect of doubling of the volume on the threshold of fragmentation processes with rotate spin or spin flip, identified in the vicinity of the masses to the giant resonance of $C E(G R)$ nucleus of type E0, E1, etc. with nucleus spin-flip within the width of the GR 4-6 MeV, as well as the values of threshold energy of 100 and $200 \mathrm{Mev} G R$ excitation, observed on all nucleus $C E$, as fully relevant nature of $G R$ and dilaton symmetry, underlying the classification $C E$ by their masses dilaton number $A$ and $Z m$ ( $m$ is the mass of an electron). $X$-structure of $C E$ set numbers of quarks and leptons $12+12$ and two dimensionless parameters - $\Theta(\sin \Theta=1 / 2.0134)$ of the mass corner of hydrogen molecules in relation to the total weight of all three generations of leptons (and are discussed) matching a numerically with the double $\alpha$ particle mass, and-the minimum number of basic types of the spinor connection in the Cartan-Weyl S = 18 (see [1]), on which the calculated mass of leptons angle $\mathrm{M}_{\mathrm{L}} /\left(2 \mathrm{M}_{\mathrm{Q}}\right)=0.2222 \ldots \ldots\left(12.7^{\circ}\right)$. Calculated resonant $\mathrm{X}$-structure is defined in the stability area of the $C E$ to Alpha decay $50<A a . u . m<.142$, and represents a number (10) of the Gaussian peaks of the width 7 a.u.m., separated by an interval of 4-5 a.u.m. (dual and triple barreled out of more narrow peaks in most cases), responsible in terms of numbers of filling ( $\mathrm{T}, \mathrm{L})$ of single-particle and hole states the shell-filled by $\mathrm{L}$ or $\mathrm{T}(\mathrm{L}, \mathrm{T}=0,1,2, \ldots$, the 12). Upper bound calculated mass $\mathrm{M}(12,12)=208.98 \mathrm{a} . u \mathrm{~m}$. is a $B i$ 208.98 completes the list of stable CE. The rest of the Po
CEare alpha-radioactive. Found that the spectral distribution of the nuclei masses variances from the resonant $\mathrm{X}$ - structure thresholds do not depend from the $C E$ masses $(A)$. Resonant peaks are characterized by abrupt doubling (recession) degree of degeneration by mass-energy in the configuration space of $\mathrm{M}(\mathrm{T}, \mathrm{L})$ masses $C E$, What characterizes the spontaneous symmetry breaking in phase transition. Doubling the probability density leads to a doubling of the size of the nucleus, which is double the size of the nucleus in the GR type E0 with spin-flip. and is exactly (with little spread 4-6 $\mathrm{MeV}$ ) to the author of the range of discrete levels, calculated by the method of quantization Dirac's spinor on the light cone near exact solution to the spontaneous breaking of symmetry in the Higgs mechanism.

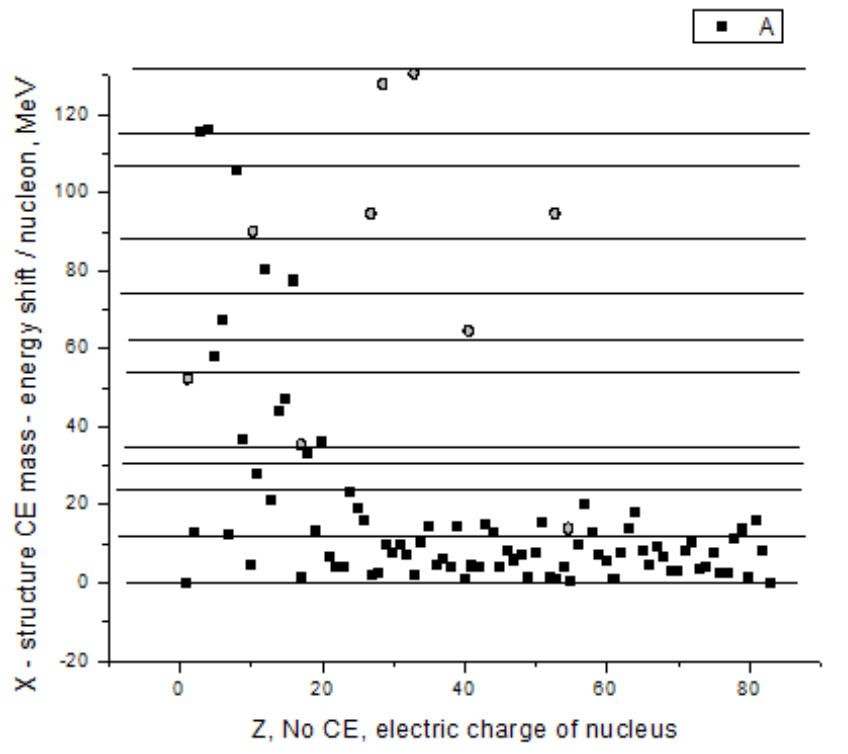

Fig. 1. Large scale-invariant (mass-charge-invariant) dependence of the mass - energy shift from the jump X-mass structure of CE on the nucleon, $\mathrm{MeV}$. Provides estimated energy levels on the light cone. Circles are full shifts for neutral atoms.

Independence from $A$, as the scale factor, mass deviation $C E$ (and especially- nuclei) from the resonant structure of $X$-thresholds means the precise execution of large-scale dilaton symmetry. All the equations for the masses $C E$ identified within the disorder on carbon C-12 (up to 5 characters) table of Mendeleev.

Standard deviation masses of chemical elements from 
jumps of quantum X-structure - offset -0.044 a.u.m. $=-41.0$ $\mathrm{MeV}$ centered on the energy coordinate of jumps within the range in $G R$ widths within a mass of a nucleon. In only two cases, CE No79 and No81 offset close to $3 \sigma$.

\section{Conclusion}

According to table 5 we can conclude from the stated some analogy between properties of hypernucleus and supernucleus with properties of elementary particles and resonances electroproduction by spin shock waves in beam ions that

1) already lightest hypernucleus kind ${ }_{\Lambda}^{2} N$ are not formed so as $\frac{m_{\Lambda}}{M_{N}}=1.3$ (No 5), there is ${ }_{\Lambda}^{3} n p$ only. In this case $\frac{m_{\Lambda}}{M_{n p}}=0.652$ is near to the ratio $\frac{m_{E P, \text { Res }}}{M_{\text {Nucleous }}}=$ $0.412 \ldots$ There are as minimum eight supernuclei for which that ratio is fulfilled too. Theoretically for all hypernucleus it very may be possible that $\Lambda$ interacts with two NN from the external level of nucleus core. For $\mathrm{He}_{4}$ in the $\tau$-atom $\frac{m_{\tau}}{M_{\mathrm{He}_{4}}}=0.475$ is near 0.41 is seen through the lepton universality as atom on the outer shell of which, instead of electron - a heavy lepton - tauon. The ions list for resonances (table 2) begin by $(n, p)$ nucleus, on the first hypernucleus decay channel - on $\mathrm{D}_{2}$.

2) There are two kind of hypernucleus ${ }_{\Omega}^{A} C E$ or ${ }_{\Omega \Omega}^{A} C E$ and supernucleus ${ }_{\Lambda}^{A} C E$ or ${ }_{\Lambda \Lambda}^{A} C E$ also as there are two groups of all-known elementary particles and resonances in electroproduction sets by means an ion electric charge doubling. Theoretically for all hypernucleus it very may be possible that $\Lambda$ interacts with two NN from the external level of nucleus core. Following the lepton universality tauons in the tau-atom will occupy the lower level closest to the nucleus, on which may be one or two particles. Hyperons mass near to the mass of the tauon, likely will also be able to hold the most one- and two particles level of the lower shell. What is in the hypernucleus case also.

3) According to data from $\gamma$ - spectroscopy [8] decays ${ }_{\Lambda}^{4} \mathrm{H}$ and ${ }_{\Lambda}^{4} \mathrm{He}$ occurs with a predominance of $\Lambda^{0}$ spin flip (full momentum of the excited P-state $\mathrm{J}=1$ ). This is consistent with the fact that in the elementary particles and resonances electro - production take place on a threshold of spin shock-waves with the electromagnetic field spin-flip.

4) Stated in [2] the fact of repeating of all-known elementary particles and resonances mass spectrum as whole of nuclei atomic weight of ions of some stable chemical elements with a coefficient in proportion to $\mathrm{M} / \mathrm{e}$ for an ion is signed that all-known elementary particles and resonances masses also as masses of stable chemical elements and isotopes are defined by positions of features - jumps of a quark - lepton dilaton $\mathrm{X}$ - structure [1]. Whereas the definition of a value of the ratio between the masses of particles and nuclei requires taking into account the contribution of SSW C-number components of classical interact.
A border of chemical elements stability through $\mathrm{Bi}-209$ is defined by possible processes of electroproductionon a threshold of spin shock-waves forming in accelerated beams of ions radioactive Po and At on a mass of $\mathrm{W}^{+}$boson of electroweak interaction.

That all gives addition arguments for observing in Veil Cartan space - time $\mathrm{V}_{4}$ of the threshold effect on GUT gravitation masses $\sim 3 \mathrm{TeV}$ for example in BAC $p \bar{p}$ collisions (see $[2,11]$ ). $3 \mathrm{TeV}-$ it is $10^{-12}$ from physical GUT masses $3 \cdot 10^{15} \mathrm{GeV}$. $10^{-12}$ gives experimental value of distinguish between gravitation and inertial masses. There we have the only possibility in experimental operating by "tales" of giant GUT masses, so that Standard Model with Conformal Gauge Theory of Gravitation $[1,11]$ is correct.

\section{References}

[1] SYROMYATNIKOV A. G. Physical effects in Conformal Gauge Theory of Gravitation (LAP Lambert Academic Publishing GmbH \& Co. KG, Saarbrucken, Germany, 2012); SYROMYATNIKOV A. G., Method of self-consistent field in non-linear dynamics problems. ("Tip. VIKA", St. Petersburg, 1993)

[2] SYROMYATNIKOV A. G. On Similarity between All-Known Elementary Particles and Resonances Mass Spectrum and Nuclear Atomic Weight. Univ. J. Phys. and Appl. 2(2) 76-79. DOI: 10.13189/ujpa.2014.02023.

[3] COLLINS P. D. B. and SQUIRES E. J., Regge poles in particle physics (Springer-Verlag, Berlin Heidelberg New York, 1968).

[4] NAKAMURA K. et al. [Particle Date Groups], J $P G$ 37, 075021 (2010) and 2011.

[5] OKUN L. B. Leptons and quarks (Nauka, Moscow) 1990. (in Russian)

[6] SYROMYATNIKOV A. G., Vestnik Sanct Peterburgskogo $\begin{array}{llllll}\text { universiteta. Ser. } 4 . \quad \text { Vip. } 2 & \text { (2012) 108-112. }\end{array}$ SYROMYATNIKOV A. G., Vestnik Sanct Peterburgskogo universiteta. Ser. 4. Vol. 2 (60) Vip. 1 (2015) 88-92.

[7] YAKAHASHI H., AHN J. K., AKIKAWA H. et al., Observation of a ${ }_{11}^{6} \mathrm{He}$ DoubleHypernucleus, Phys. Rev. Lett. 87 No. 21 (2001) 212502.

[8] DONCHEV A. G., KALACHEV S. A., KOLESNIKOV N. N. et al., Rus.Nuclear physics. 67 No. 12 (2004) 2178-2189.

[9] NEUDATCHINV. G., OBUKHOVSKII I. T., SMIRNOV Y. F., A nonrelativistic potential model with forbidden states for the $\mathrm{n}$ - $\mathrm{n}$ interaction at small distance, Phys. Rev. Lett. B. 43 (1973) 13-16.

[10] Observation of an Antimatter Hypernucleus. The STAR Collaboration. Science 2 April 2010: 58-62. Published online 4 March 2010 [DOI: 10.1126/science.1183980].

[11] SYROMYATNIKOV A. G. On some feature of possible torsion effects on observables at hadron colliders, Int. J. Geom. Meth. Mod. Phys (2015) DOI: 10.1142/S0219887815500802. 